\title{
Expectation Consistent Approximate Inference: Generalizations and Convergence
}

\author{
Alyson K. Fletcher, Member, IEEE, Mojtaba Sahraee-Ardakan, Student Member, IEEE, \\ Sundeep Rangan, Fellow, IEEE, and Philip Schniter, Fellow, IEEE
}

\begin{abstract}
Approximations of loopy belief propagation, including expectation propagation and approximate message passing, have attracted considerable attention for probabilistic inference problems. This paper proposes and analyzes a generalization of Opper and Winther's expectation consistent (EC) approximate inference method. The proposed method, called Generalized Expectation Consistency (GEC), can be applied to both maximum a posteriori (MAP) and minimum mean squared error (MMSE) estimation. Here we characterize its fixed points, convergence, and performance relative to the replica prediction of optimality.
\end{abstract}

Index Terms-Expectation propagation, Approximate message passing, Bethe free energy, S-transform in free probability

\section{INTRODUCTION}

Consider the problem of estimating a random vector $\mathbf{x} \in$ $\mathbb{R}^{N}$ from observations $\mathbf{y} \in \mathbb{R}^{M}$ under the posterior density

$$
p(\mathbf{x} \mid \mathbf{y})=Z^{-1} \exp \left[-f_{1}(\mathbf{x})-f_{2}(\mathbf{x})\right],
$$

where $Z=\int \exp \left[-f_{1}(\mathbf{x})-f_{2}(\mathbf{x})\right] \mathrm{d} \mathbf{x}$ is a normalization constantsometimes called the partition function and $f_{i}(\mathbf{x})$ are penalty functions. Although both $Z$ and the penalties $f_{i}$ may depend on $\mathbf{y}$, our notation suppresses this dependence. We are interested in two problems:

- MAP estimation: In maximum a posteriori (MAP) estimation, we wish to find the point estimate $\widehat{\mathbf{x}}=$ $\arg \max _{\mathbf{x}} p(\mathbf{x} \mid \mathbf{y})$, equivalently stated as

$$
\widehat{\mathbf{x}}=\underset{\mathbf{x}}{\arg \min }\left[f_{1}(\mathbf{x})+f_{2}(\mathbf{x})\right] .
$$

- MMSE estimation and approximate inference: In minimum mean-squared error (MMSE) estimation, we wish to compute the posterior mean $\mathbb{E}(\mathbf{x} \mid \mathbf{y})$ and maybe also approximations of the posterior covariance $\operatorname{Cov}(\mathbf{x} \mid \mathbf{y})$ or marginal posterior densities $\left\{p\left(x_{n} \mid \mathbf{y}\right)\right\}_{n=1}^{N}$.

For the MAP estimation problem (2), the separable structure of the objective function can be exploited by one of several optimization methods, including variants of the iterative

A. Fletcher and M. Saharee (email: akfletcher@ucla.edu, msahraee@ucsc.edu) are with the Department of Statistics and Electrical Engineering, University of California, Los Angeles. Their work is supported in part by the National Science Foundation under Grant No. 1254204 and the Office of Naval Research Grant No. N00014-15-1-2677.

S. Rangan (email: srangan@nyu.edu) is with the Department of Electrical and Computer Engineering, New York University, Brooklyn, NY. His work was supported by the National Science Foundation under Grant No. 1116589 and the industrial affiliates of NYU WIRELESS.

P. Schniter (email: schniter@ece.osu.edu) is with the Department of Electrical and Computer Engineering, The Ohio State University. His work was supported in part by the National Science Foundation under Grants CCF1218754 and CCF-1527162. shrinkage and thresholding algorithm (ISTA) [1] , [2]-[6] and the alternating direction method of multipliers (ADMM) [7] , [8]-[10].

The MMSE and inference problems, however, are more difficult [11], even for the case of convex penalties [12], [13]. In recent years, there has been considerable interest in approximations of loopy belief propagation [14], [15] for both MMSE estimation and approximate inference. These methods include variants of expectation propagation (EP) [16]-[18] and, more recently, approximate message passing (AMP) [19], [20], [21]-[23]. For a posterior of the form (1), both EP and AMP reduce the inference problem to a sequence of problems involving only one penalty at a time. These "local" problems are computationally tractable under suitable penalties. Moreover, in certain large random instances, these methods are provably optimal [24], [20], [25]. Due to their generality, these methods have been successfully applied to a wide range of problems, e.g., [26], [27], [27]-[33].

Despite their computational simplicity, the convergence and accuracy of these methods are not fully understood. This work analyzes one promising EP-type method known as expectation consistent approximate inference (EC), originally proposed by Opper and Winther in [17]. As shown in [18], EC interpreted as a parallel form of the EP method from [16], while being closely related to the adaptive TAP method from [34] [35].

As we now describe, our work contributes to the extension and understanding of Opper and Winther's EC method.

- Generalization: We propose and analyze a generalization of the EC algorithm that we call Generalized EC (GEC). The proposed method can be applied to arbitrary penalties $f_{1}(\mathbf{x})$ and $f_{2}(\mathbf{x})$, and can also be used for both MAP or MMSE inference by appropriate selection of estimation functions. Standard EC typically applies only to MMSE inference, often with one penalty being quadratic. Also, GEC supports a generalization of the covariance diagonalization step, which is one of the key computational bottlenecks in standard EC [12].

- Fixed points: It is well known that, when the standard EC algorithm converges, its fixed points can be interpreted as saddle points of an energy function [17], [18] similar to the Bethe Free Energy (BFE) that arises in the analysis of loopy BP [15]. We provide a similar energy-function interpretation of the MMSE-GEC algorithm (Theorem 3). Our analysis shows that the so-called first- and secondorder terms output by MMSE-GEC can be interpreted as estimates of the posterior mean and variance. Regarding the fixed points of MAP-GEC, we show that the first- 
order terms are critical points of the objective function (2) and the second-order terms can be interpreted as estimates of the local curvature of the objective function.

- Convergence: A critical concern for both EP and AMP is convergence [12], [36] [37], [38]. This situation is perhaps not surprising, given that they derive from loopy $\mathrm{BP}$, which also may diverge. Most provably convergent alternate approaches are based on variants of doubleloop methods such as [13], [17]. Other modifications to improve the stability include damping and fractional updates [36], [39], [40] and sequential updating [41], which increase the likelihood of convergence at the cost of convergence speed. Our analysis of GEC convergence considers the first- and second-order terms separately-a decoupling technique also used in [18], [42]. We show that, for strictly convex, smooth penalties, the standard updates for the first-order terms are provably convergent. For MAP-GEC, the second-order terms converge as well.

- Relation to the replica prediction of optimality: In [43], Tulino et al. used a replica analysis from statistical physics to predict the the MMSE error when estimating a random vector $\mathrm{x}$ from noisy measurements of the linear transformation $\mathbf{A x}$ under large, unitarily invariant, random $\mathbf{A}$. This work extended the replica analyses in [44]-[46], which applied to i.i.d. A. (See also [47].) In [48], [49], Çakmak et al. proposed a variant of AMP (called S-AMP) using closely related methods. In this work, we show that, when GEC is applied to linear regression, a prediction of the posterior MSE satisfies a fixed point equation that exactly matches the replica prediction from [43].

- Relation to ADMM: ADMM [7] is a popular approach to optimization problems of the form (2) with convex $f_{i}$. Essentially, ADMM iterates individual optimizations of $f_{1}$ and $f_{2}$ together with a "dual update" that (asymptotically) enforces consistency between the individual optimizations. The dual update involves a fixed step size, whose choice affects the convergence speed of the algorithm. In this work, we show that GEC can be interpreted as a variant of ADMM with two dual-updates, each with a step size that is adapted according to the local curvature of the corresponding penalty $f_{i}$.

\section{The Generalized EC Algorithm}

\section{A. Estimation and Diagonalization}

The proposed GEC algorithm involves two key operations: i) estimation, which computes an estimate of $\mathrm{x}$ using one penalty at a time; and ii) diagonalization of a sensitivity term.

Estimation: The estimation function is constructed differently for the MAP and MMSE cases. In the MAP case, the estimation function is given by

$$
\mathbf{g}_{i}\left(\mathbf{r}_{i}, \boldsymbol{\gamma}_{i}\right):=\underset{\mathbf{x}}{\arg \min }\left[f_{i}(\mathbf{x})+\frac{1}{2}\left\|\mathbf{x}-\mathbf{r}_{i}\right\|_{\boldsymbol{\gamma}_{i}}^{2}\right],
$$

where $\mathbf{r}_{i}, \gamma_{i} \in \mathbb{R}^{N}$ and $\gamma_{i}>0$ (componentwise), and where

$$
\|\mathbf{v}\|_{\gamma}^{2}:=\sum_{n=1}^{N} \gamma_{n}\left|v_{n}\right|^{2}
$$

for any $\mathbf{v}$ and positive $\gamma$. The estimation function (3) is a scaled version of what is often called the proximal operator.

For the MMSE problem, the estimation function is

$$
\mathbf{g}_{i}\left(\mathbf{r}_{i}, \gamma_{i}\right):=\mathbb{E}\left[\mathbf{x} \mid \mathbf{r}_{i}, \gamma_{i}\right],
$$

where the expectation is with respect to the conditional density

$$
p_{i}\left(\mathbf{x} \mid \mathbf{r}_{i}, \gamma_{i}\right)=\frac{1}{Z_{i}} \exp \left[-f_{i}(\mathbf{x})-\frac{1}{2}\left\|\mathbf{x}-\mathbf{r}_{i}\right\|_{\gamma_{i}}^{2}\right] .
$$

Diagonalization: In its more general form, the diagonalization operator $\mathbf{d}(\mathbf{Q})$ is an affine linear map from $\mathbf{Q} \in \mathbb{R}^{N \times N}$ to $\mathbb{R}^{N}$. Several instances of diagonalization are relevant to our work. For example, vector-valued diagonalization,

$$
\mathbf{d}(\mathbf{Q}):=\operatorname{diag}(\mathbf{Q}),
$$

which simply returns a $N$-dimensional vector containing the diagonal elements of $\mathbf{Q}$, and uniform diagonalization,

$$
\mathbf{d}(\mathbf{Q}):=N^{-1} \operatorname{tr}(\mathbf{Q}) \mathbf{1}_{N},
$$

which returns a constant vector containing the average diagonal element of $\mathbf{Q}$. Here, $\mathbf{1}_{N}$ denotes the $N$-dimensional vector with all elements equal to one.

For the separable GLM, it will be useful to consider a block uniform diagonalization. In this case, we partition

$$
\mathbf{x}=\left(\mathbf{x}_{1} ; \cdots ; \mathbf{x}_{L}\right), \quad \mathbf{x}_{\ell} \in \mathbb{R}^{N_{\ell}},
$$

with $\sum_{\ell} N_{\ell}=N$. Conformal to the partition, we define the block uniform diagonalization

$$
\mathbf{d}(\mathbf{Q}):=\left(d_{1} \mathbf{1}_{N_{1}} ; \cdots ; d_{L} \mathbf{1}_{N_{L}}\right), \quad d_{\ell}=\frac{1}{N_{\ell}} \operatorname{tr}\left(\mathbf{Q}_{\ell \ell}\right),
$$

where $\mathbf{Q}_{\ell \ell} \in \mathbb{R}^{N_{\ell} \times N_{\ell}}$ is the $\ell$-th diagonal block of $\mathbf{Q}$.

We note that any of these diagonalization operators can be used with either the MAP or MMSE estimation functions.

\section{B. Algorithm Description}

The generalized EC (GEC) algorithm is specified in Algorithm 1. There, $\partial \mathbf{g}_{i}\left(\mathbf{r}_{i}, \boldsymbol{\gamma}_{i}\right) / \partial \mathbf{r}_{i}$ is the $N \times N$ Jacobian matrix of $\mathbf{g}_{i}$ evaluated at $\left(\mathbf{r}_{i}, \gamma_{i}\right), \operatorname{Diag}\left(\boldsymbol{\gamma}_{i}\right)$ is the diagonal matrix whose diagonal equals $\gamma_{i}$, "./" is componentwise vector division, and "." is componentwise vector multiplication. Note that it is not necessary to compute the full matrix $\mathbf{Q}_{i}$ in line 5 , it suffices to compute only the diagonalization $\mathbf{d}\left(\mathbf{Q}_{i}\right)$.

It will sometimes be useful to rewrite Algorithm 1 in a scaled form. Define $\boldsymbol{\beta}_{i}:=\boldsymbol{\gamma}_{i} \cdot \mathbf{r}_{i}$ and $\widetilde{\mathbf{g}}_{i}\left(\boldsymbol{\beta}_{i}, \boldsymbol{\gamma}_{i}\right):=$ $\mathbf{g}_{i}\left(\boldsymbol{\beta}_{i} \cdot / \boldsymbol{\gamma}_{i}, \boldsymbol{\gamma}_{i}\right)$. Then GEC can be rewritten as

$$
\begin{aligned}
\boldsymbol{\eta}_{i} & \leftarrow \mathbf{1 .} / \mathbf{d}\left(\widetilde{\mathbf{Q}}_{i}\right), \widetilde{\mathbf{Q}}_{i}:=\partial \widetilde{\mathbf{g}}_{i}\left(\boldsymbol{\beta}_{i}, \gamma_{i}\right) / \partial \boldsymbol{\beta}_{i} \\
\gamma_{j} & \leftarrow \boldsymbol{\eta}_{i}-\gamma_{i} \\
\boldsymbol{\beta}_{j} & \leftarrow \boldsymbol{\eta}_{i} \cdot \widetilde{\mathbf{g}}_{i}\left(\boldsymbol{\beta}_{i}, \boldsymbol{\gamma}_{i}\right)-\boldsymbol{\beta}_{i} .
\end{aligned}
$$

Note that, in line 5] of Algorithm 1] we are required to compute the (scaled) Jacobian of the estimation function. For the MAP estimation function (3), this quantity becomes [20]

$$
\left[\partial \mathbf{g}_{i}\left(\mathbf{r}_{i}, \boldsymbol{\gamma}_{i}\right) / \partial \mathbf{r}_{i}\right] \boldsymbol{\Gamma}_{i}^{-1}=\left[\mathcal{H}_{f_{i}}\left(\widehat{\mathbf{x}}_{i}\right)+\boldsymbol{\Gamma}_{i}\right]^{-1},
$$




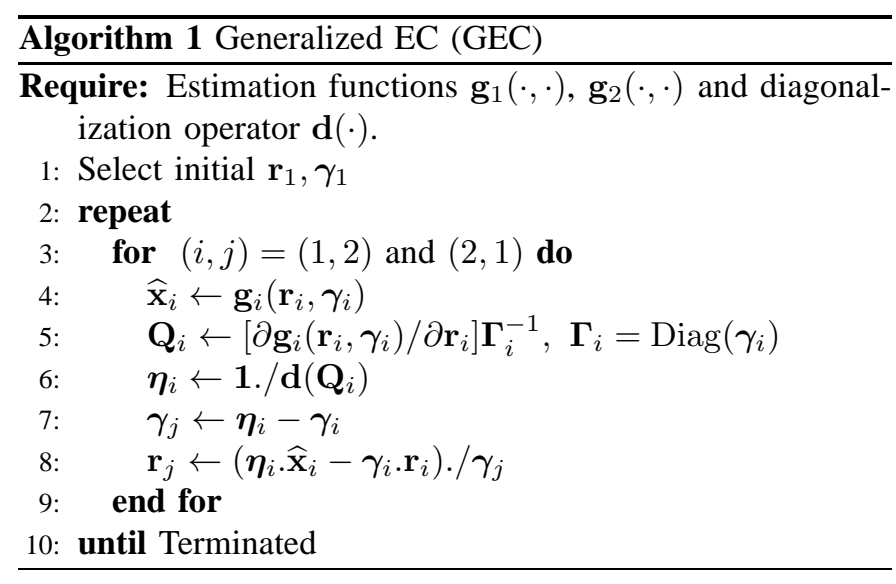

where $\widehat{\mathbf{x}}_{i}$ is the minimizer in (3) and $\mathcal{H}_{f_{i}}\left(\widehat{\mathbf{x}}_{i}\right)$ is the Hessian of $f_{i}$ at that minimizer. For the MMSE estimation function, this scaled Jacobian becomes the covariance matrix

$$
\left[\partial \mathbf{g}_{i}\left(\mathbf{r}_{i}, \gamma_{i}\right) / \partial \mathbf{r}_{i}\right] \boldsymbol{\Gamma}_{i}^{-1}=\operatorname{Cov}\left(\mathbf{x}_{i} \mid \mathbf{r}_{i}, \gamma_{i}\right)
$$

where the covariance is taken with respect to the density (5).

\section{Examples}

SLR with Separable Prior: Suppose that we aim to estimate $\mathbf{x}$ given noisy linear measurements of the form

$$
\mathbf{y}=\mathbf{A} \mathbf{x}+\mathbf{w}, \quad \mathbf{w} \sim \mathcal{N}\left(0, \gamma_{w}^{-1} \mathbf{I}\right),
$$

where $\mathbf{A} \in \mathbb{R}^{M \times N}$ is a known matrix and $\mathbf{w}$ is independent of $\mathbf{x}$. Statisticians often refer to this problem as standard linear regression (SLR). Suppose also that $\mathbf{x}$ has independent elements with marginal densities $p\left(x_{n}\right)$ :

$$
p(\mathbf{x})=\prod_{n=1}^{N} p\left(x_{n}\right)
$$

Then, the posterior $p(\mathbf{x} \mid \mathbf{y})$ takes the form of (1) when

$$
f_{1}(\mathbf{x})=-\sum_{n=1}^{N} \log p\left(x_{n}\right), \quad f_{2}(\mathbf{x})=\frac{\gamma_{w}}{2}\|\mathbf{y}-\mathbf{A} \mathbf{x}\|^{2} .
$$

The separable nature of $f_{1}(\mathbf{x})$ implies that, in both the MAP or MMSE cases, the output of the estimator $g_{1}$ (recall (3) and (4)) can be computed in a componentwise manner, as can the diagonal terms of their Jacobians. Likewise, the quadratic nature of $f_{2}(\mathbf{x})$ implies that the output of $\mathbf{g}_{2}$ can be computed by solving a linear system.

GLM with Separable Prior: Now suppose that, instead of (13), we have a more general likelihood with the form

$$
p(\mathbf{y} \mid \mathbf{x})=\prod_{m=1}^{M} p\left(y_{m} \mid z_{m}\right), \quad \mathbf{z}=\mathbf{A} \mathbf{x} .
$$

Statisticians often refer to (16) as the generalized linear model (GLM) [50], [51]. To pose the GLM in a format convenient for GEC, we define the new vector $\mathbf{u}=(\mathbf{x} ; \mathbf{z})$. Then, the posterior $p(\mathbf{u} \mid \mathbf{y})=p(\mathbf{x}, \mathbf{z} \mid \mathbf{y})$ can be placed in the form of (1) using the penalties

$$
\begin{aligned}
& f_{1}(\mathbf{u})=f_{1}(\mathbf{x}, \mathbf{z})=-\sum_{n=1}^{N} \log p\left(x_{n}\right)-\sum_{m=1}^{M} \log p\left(y_{m} \mid z_{m}\right), \\
& f_{2}(\mathbf{u})=f_{2}(\mathbf{x}, \mathbf{z})= \begin{cases}0 & \text { if } \mathbf{z}=\mathbf{A x}, \\
\infty & \text { if } \mathbf{z} \neq \mathbf{A} \mathbf{x},\end{cases}
\end{aligned}
$$

where $f_{2}(\mathbf{u})$ constrains $\mathbf{u}$ to the nullspace of $[\mathbf{A}-\mathbf{I}]$. Because the first penalty $f_{1}(\mathbf{u})$ remains separable, the MAP and MMSE functions can be evaluated componentwise, as in separable SLR. For the second penalty $f_{2}(\mathbf{u})$, MAP or MMSE estimation simply becomes projection onto a linear space.

\section{FIXED PoInTs of GEC}

\section{A. Consistency}

We now characterize the fixed points of GEC for both MAP and MMSE estimation functions. For both scenarios, we will need the following simple consistency result.

Lemma 1. Consider GEC (Algorithm 1) with arbitrary estimation functions $\mathbf{g}_{i}(\cdot, \cdot)$ and arbitrary diagonalization operator $\mathrm{d}(\cdot)$. For any fixed point with $\gamma_{1}+\gamma_{2}>0$, we have

$$
\begin{aligned}
& \boldsymbol{\eta}_{1}=\boldsymbol{\eta}_{2}=\boldsymbol{\eta}:=\gamma_{1}+\gamma_{2} \\
& \widehat{\mathbf{x}}_{1}=\widehat{\mathbf{x}}_{2}=\widehat{\mathbf{x}}:=\left(\gamma_{1} \mathbf{r}_{1}+\gamma_{2} \mathbf{r}_{2}\right) \cdot /\left(\gamma_{1}+\gamma_{2}\right)
\end{aligned}
$$

Proof: From line 7 of Algorithm 1, $\boldsymbol{\eta}_{i}=\gamma_{1}+\gamma_{2}$ for $i=1,2$, which proves 118a). Also, since $\gamma_{1}+\gamma_{2}>0$, the elements of $\boldsymbol{\eta}$ are invertible. In addition, from line 8

$$
\widehat{\mathbf{x}}_{i}=\left(\boldsymbol{\gamma}_{1} \cdot \mathbf{r}_{1}+\boldsymbol{\gamma}_{2} \cdot \mathbf{r}_{2}\right) \cdot / \boldsymbol{\eta}_{i} \text { for } i=1,2,
$$

which proves $18 \mathrm{~b}$.

\section{B. MAP Estimation}

We first examine GEC's fixed points for MAP estimation.

Theorem 1. Consider GEC (Algorithm 1) with the MAP estimation functions from (3) and an arbitrary diagonalization $\mathbf{d}(\cdot)$. For any fixed point with $\gamma_{i}>0$, let $\widehat{\mathbf{x}}=\widehat{\mathbf{x}}_{i}$ be the common value of the two estimates as defined in Lemma 1 Then $\widehat{\mathbf{x}}$ is a stationary point of the minimization (2).

\section{Proof: See Appendix A}

\section{MAP Estimation and Curvature}

Note that Theorem 1 applies to an arbitrary diagonalization operator $\mathbf{d}(\cdot)$. This raises two questions: i) what is the role of the diagonalization operator $\mathbf{d}(\cdot)$, and ii) how can the fixed point $\boldsymbol{\eta}$ be interpreted as a result of that diagonalization? We now show that, under certain additional conditions and certain choices of $\mathbf{d}, \boldsymbol{\eta}$ can be related to the curvature of the optimization objective in (2).

Let $\widehat{\mathbf{x}}$ be a stationary point of (2) and let $\mathbf{P}_{i}=\mathcal{H}_{f_{i}}(\widehat{\mathbf{x}})$ be the Hessian of $f_{i}$ at $\widehat{\mathbf{x}}$. Then, the Hessian of the objective function in (2) is $\mathbf{P}_{1}+\mathbf{P}_{2}$. Furthermore, let

$$
\widehat{\boldsymbol{\eta}}:=\mathbf{1} \cdot / \mathbf{d}\left(\left(\mathbf{P}_{1}+\mathbf{P}_{2}\right)^{-1}\right),
$$


so that $1 . / \widehat{\eta}$ is the diagonal of the inverse Hessian. Geometrically speaking, this inverse Hessian measures the curvature of the objective function at the critical point $\widehat{\mathbf{x}}$.

We now identify two cases where $\boldsymbol{\eta}=\widehat{\boldsymbol{\eta}}$ : i) when $\mathbf{P}_{i}$ are diagonal, and ii) when $\mathbf{P}_{i}$ are free. To define "free," consider the Stieltjes transform $S_{\mathbf{P}}(\omega)$ of any real symmetric matrix $\mathbf{P}$ :

$$
S_{\mathbf{P}}(\omega)=\frac{1}{N} \operatorname{tr}\left[(\mathbf{P}-\omega \mathbf{I})^{-1}\right]=\frac{1}{N} \sum_{n=1}^{N} \frac{1}{\lambda_{n}-\omega},
$$

where $\lambda_{n}$ are the eigenvalues of $\mathbf{P}$. Also, let $R_{\mathbf{P}}(\omega)$ denote the so-called $R$-transform of $\mathbf{P}$, given by

$$
R_{\mathbf{P}}(\omega)=S_{\mathbf{P}}^{-1}(-\omega)-\frac{1}{\omega},
$$

where the inverse $S_{\mathbf{P}}^{-1}(\cdot)$ is in terms of composition of functions. The Stieltjes and $R$-transforms are discussed in detail in [52]. We will say that $\mathbf{P}_{1}$ and $\mathbf{P}_{2}$ are "free" if

$$
R_{\mathbf{P}_{1}+\mathbf{P}_{2}}(\omega)=R_{\mathbf{P}_{1}}(\omega)+R_{\mathbf{P}_{2}}(\omega) .
$$

An important example of freeness is the following. Suppose that the penalty functions are given by $f_{i}(\mathbf{x})=h_{i}\left(\mathbf{A}_{i} \mathbf{x}\right)$ for some matrices $\mathbf{A}_{i}$ and functions $h_{i}(\cdot)$. Then

$$
\mathbf{P}_{i}=\mathcal{H}_{f_{i}}(\widehat{\mathbf{x}})=\mathbf{A}_{i}^{\top} \mathcal{H}_{h_{i}}\left(\widehat{\mathbf{z}}_{i}\right) \mathbf{A}_{i}, \quad \widehat{\mathbf{z}}_{i}=\mathbf{A}_{i} \widehat{\mathbf{x}} .
$$

It is shown in [52] that, if $\widehat{\mathbf{z}}_{i}$ are fixed and $\mathbf{A}_{i}$ are unitarily invariant random matrices, then $\mathbf{P}_{i}$ are asymptotically free in certain limits as $N \rightarrow \infty$. Freeness will thus occur in the limits of large problem with unitarily invariant random matrices.

Theorem 2. Consider GEC (Algorithm 1) with the MAP estimation functions (3). Consider any fixed point with $\gamma_{i}>0$, and let $\widehat{\mathbf{x}}$ and $\boldsymbol{\eta}$ be the common values of $\widehat{\mathbf{x}}_{i}$ and $\boldsymbol{\eta}_{i}$ from Lemma 1] Recall that $\widehat{\mathbf{x}}$ is a stationary point of the minimization (2) via Theorem 1 Then $\boldsymbol{\eta}=\widehat{\boldsymbol{\eta}}$ from (19) under either

(a) vector-valued $\mathbf{d}(\cdot)$ from (6) and diagonal $\mathbf{P}_{i}$; or

(b) uniform $\mathbf{d}(\cdot)$ from (7) and free $\mathbf{P}_{i}$.

Proof: See Appendix B

\section{MMSE Estimation}

We now consider the fixed points of GEC under MMSE estimation functions. It is well-known that the fixed points of the standard EC algorithm are critical points of a certain freeenergy optimization for approximate inference [17], [18]. We derive a similar characterization of the fixed points of GEC.

Let $p(\mathbf{x} \mid \mathbf{y})$ be the density (1) for some fixed $\mathbf{y}$. Then, given any density $b(\mathbf{x})$, it is straightforward to show that the KL divergence between $b(\mathbf{x})$ and $p(\mathbf{x} \mid \mathbf{y})$ can be expressed as

$$
D(b \| p)=D\left(b \| e^{-f_{1}}\right)+D\left(b \| e^{-f_{2}}\right)+H(b)+\text { const },
$$

where $H(b)$ is the differential entropy of $b$ and the constant term does not depend on $b$. Thus, in principle, we could compute $p$ by minimizing (23) over all densities $b$. Of course, this minimization is generally intractable since it involves a search over an $N$-dimensional density.

To approximate the minimization, define

$$
J\left(b_{1}, b_{2}, q\right):=D\left(b_{1} \| e^{-f_{1}}\right)+D\left(b_{2} \| e^{-f_{2}}\right)+H(q),
$$

where $b_{1}, b_{2}$ and $q$ are densities on the variable $\mathbf{x}$. Note that minimization of (23) over $b$ is equivalent to the optimization

$$
\min _{b_{1}, b_{2}} \max _{q} J\left(b_{1}, b_{2}, q\right)
$$

under the constraint

$$
b_{1}=b_{2}=q .
$$

The energy function (24) is known as the Bethe Free Energy (BFE). Under the constraint (26), the BFE matches the original energy function (23). However, BFE minimization under the constraint (26) is equally intractable.

As with EC, the GEC algorithm can be derived as a relaxation of the above BFE optimization, wherein (26) is replaced by the so-called moment matching constraints:

$$
\begin{aligned}
\mathbb{E}\left(\mathbf{x} \mid b_{1}\right) & =\mathbb{E}\left(\mathbf{x} \mid b_{2}\right)=\mathbb{E}(\mathbf{x} \mid q) \\
\mathbf{d}\left(\mathbb{E}\left(\mathbf{x} \mathbf{x}^{\top} \mid b_{1}\right)\right) & =\mathbf{d}\left(\mathbb{E}\left(\mathbf{x} \mathbf{x}^{\top} \mid b_{2}\right)\right)=\mathbf{d}\left(\mathbb{E}\left(\mathbf{x x}^{\top} \mid q\right)\right) .
\end{aligned}
$$

Thus, instead of requiring a perfect match in the densities $b_{1}, b_{2}, q$ as in 26, GEC requires only a match in their first moments and certain diagonal components of their second moments. Note that, for the vector-valued diagonalization (6), (27b) is equivalent to

$$
\mathbb{E}\left[x_{n}^{2} \mid b_{i}\right]=\mathbb{E}\left[x_{n}^{2} \mid q\right] \quad \forall n, i,
$$

which requires only that the marginal 2 nd moments match. Under the uniform diagonalization (7), (27b) is equivalent to

$$
\frac{1}{N} \sum_{n=1}^{N} \mathbb{E}\left[x_{n}^{2} \mid b_{i}\right]=\frac{1}{N} \sum_{n=1}^{N} \mathbb{E}\left[x_{n}^{2} \mid q\right], \quad i=1,2,
$$

requiring only that the average 2 nd marginal moments match.

Theorem 3. Consider GEC (Algorithm 1) with the MMSE estimation functions (4) and either vector-valued (6) or uniform (7) diagonalization. For any fixed point with $\gamma_{i}>0$, let $\widehat{\mathbf{x}}$ and $\boldsymbol{\eta}$ be the common values of $\widehat{\mathbf{x}}_{i}$ and $\boldsymbol{\eta}_{i}$ from Lemma 1 Also let

$$
b_{i}(\mathbf{x})=p_{i}\left(\mathbf{x} \mid \mathbf{r}_{i}, \gamma_{i}\right)
$$

for $p_{i}\left(\mathbf{x} \mid \mathbf{r}_{i}, \gamma_{i}\right)$ from (5) and let $q(\mathbf{x})$ be the Gaussian density

$$
q(\mathbf{x}) \propto \exp \left[-\frac{1}{2}\|\mathbf{x}-\widehat{\mathbf{x}}\|_{\boldsymbol{\eta}}^{2}\right]
$$

Then, $b_{1}, b_{2}, q$ are stationary points of the optimization 25 subject to the moment matching constraints 27. In addition, $\widehat{\mathbf{x}}$ is the mean, and $\boldsymbol{\eta}$ the marginal precision, of these densities:

$$
\begin{aligned}
\widehat{\mathbf{x}} & =\mathbb{E}(\mathbf{x} \mid q)=\mathbb{E}\left(\mathbf{x} \mid b_{i}\right), i=1,2 \\
\mathbf{1 .} / \boldsymbol{\eta} & =\mathbf{d}\left(\operatorname{Cov}\left(\mathbf{x} \mathbf{x}^{\top} \mid q\right)\right)=\mathbf{d}\left(\operatorname{Cov}\left(\mathbf{x} \mathbf{x}^{\top} \mid b_{i}\right)\right), i=1,2 .
\end{aligned}
$$

Proof: See Appendix C.

\section{E. An Unbiased Estimate of $\mathrm{x}$}

As described in Section I-C, a popular application of GEC is to approximate the marginals of the posterior density (1) in the case that the first penalty $f_{1}(\mathbf{x})$ describes the prior and the second penalty $f_{2}(\mathbf{x} ; \mathbf{y})$ describes the likelihood. That is,

$$
p(\mathbf{x}) \propto e^{-f_{1}(\mathbf{x})} \text { and } p(\mathbf{y} \mid \mathbf{x}) \propto e^{-f_{2}(\mathbf{x} ; \mathbf{y})} .
$$


Here, we have made the dependence of $f_{2}(\mathbf{x} ; \mathbf{y})$ on $\mathbf{y}$ explicit. The GEC algorithm produces three estimates for the posterior density: $b_{1}, b_{2}$, and $q$. Consider the first of these estimates, $b_{1}$. From (28) and (5), this belief estimate is given by

$$
b_{1}\left(\mathbf{x} ; \mathbf{r}_{1}, \boldsymbol{\gamma}_{1}\right)=Z\left(\mathbf{r}_{1}\right)^{-1} p(\mathbf{x}) \exp \left[-\frac{1}{2}\left\|\mathbf{x}-\mathbf{r}_{1}\right\|_{\boldsymbol{\gamma}_{1}}^{2}\right] \text {, }
$$

where $Z\left(\mathbf{r}_{1}\right)$ is a normalization constant.

If we model $\mathbf{r}_{1}$ as a random vector, then (32) implies that

$$
p\left(\mathbf{x} \mid \mathbf{r}_{1}\right)=b_{1}\left(\mathbf{x} ; \mathbf{r}_{1}, \gamma_{1}\right)
$$

From Bayes rule, we know $p\left(\mathbf{x} \mid \mathbf{r}_{1}\right)=p(\mathbf{x}) p\left(\mathbf{r}_{1} \mid \mathbf{x}\right) / p\left(\mathbf{r}_{1}\right)$, which together with (32) implies

$$
p\left(\mathbf{r}_{1} \mid \mathbf{x}\right)=\frac{p\left(\mathbf{r}_{1}\right)}{Z\left(\mathbf{r}_{1}\right)} \exp \left[-\frac{1}{2}\left\|\mathbf{r}_{1}-\mathbf{x}\right\|_{\gamma_{1}}^{2}\right] .
$$

For $p\left(\mathbf{r}_{1}\right)$ to be an admissible prior density on $\mathbf{r}_{1}$, it must satisfy $p\left(\mathbf{r}_{1}\right) \geq 0, \int p\left(\mathbf{r}_{1}\right) \mathrm{d} \mathbf{r}_{1}=1$, and $\int p\left(\mathbf{r}_{1} \mid \mathbf{x}\right) \mathrm{d} \mathbf{r}_{1}=1 \forall \mathbf{x}$. It is straightforward to show that one admissible choice is

$$
p\left(\mathbf{r}_{1}\right)=c Z\left(\mathbf{r}_{1}\right), \quad c^{2}=(2 \pi)^{-N} \prod_{n=1}^{N} \gamma_{1 n} .
$$

Under this choice, we get

$$
p\left(\mathbf{r}_{1} \mid \mathbf{x}\right)=\mathcal{N}\left(\mathbf{x}, \boldsymbol{\Gamma}_{1}^{-1}\right),
$$

in which case $\mathbf{r}_{1}$ can be interpreted as an unbiased estimate of $\mathbf{x}$ with $\boldsymbol{\Gamma}_{1}^{-1}$-covariance Gaussian estimation error.

The situation above is reminiscent of AMP algorithms [19], [20]. Specifically, their state evolution analyses [24] show that, under large i.i.d. $\mathbf{A}$, they recursively produce a sequence of vectors $\left\{\mathbf{r}^{k}\right\}_{k>0}$ that can be modeled as realizations of the true vector $\mathbf{x}$ plus zero-mean white Gaussian noise. They then compute a sequence of estimates of $\mathbf{x}$ by "denoising" each $\mathbf{r}^{k}$.

\section{Convergence of the First-Order Terms For Strictly Convex Penalties}

We first analyze the convergence of GEC with fixed "second-order terms" $\boldsymbol{\eta}_{i}$ and $\gamma_{i}$. To this end, fix $\gamma_{i}>0$ at arbitrary values and assume that $\boldsymbol{\eta}_{i}$ are fixed points of 10b. Then Lemma 1 implies that $\boldsymbol{\eta}_{1}=\boldsymbol{\eta}_{2}=\boldsymbol{\eta}:=\gamma_{1}+\gamma_{2}$. With $\boldsymbol{\eta}_{i}$ and $\gamma_{i}$ fixed, the (scaled) GEC algorithm (10) updates only $\boldsymbol{\beta}_{i}=\gamma_{i} \cdot \mathbf{x}_{i}$. In particular, 10c implies that this update is

$$
\boldsymbol{\beta}_{j} \leftarrow\left(\boldsymbol{\Gamma}_{1}+\boldsymbol{\Gamma}_{2}\right) \widetilde{\mathbf{g}}_{i}\left(\boldsymbol{\beta}_{i}, \boldsymbol{\gamma}_{i}\right)-\boldsymbol{\beta}_{i},(i, j) \in\{(1,2),(2,1)\} .
$$

We analyze the recursion (34) under the following assumption

Assumption 1. For $i=1,2$, fix $\gamma_{i}>0$ and suppose that $\widetilde{\mathbf{g}}_{i}\left(\boldsymbol{\beta}_{i}, \boldsymbol{\gamma}_{i}\right)$ is differentiable in $\boldsymbol{\beta}_{i}$. Also define

$$
\widetilde{\mathbf{Q}}_{i}\left(\boldsymbol{\beta}_{i}\right):=\partial \widetilde{\mathbf{g}}_{i}\left(\boldsymbol{\beta}_{i}, \boldsymbol{\gamma}_{i}\right) / \partial \boldsymbol{\beta}_{i},
$$

and assume that $\widetilde{\mathbf{Q}}_{i}\left(\boldsymbol{\beta}_{i}\right)$ is symmetric and that there exists constants $c_{i 1}, c_{i 2}>0$ such that, for all $\boldsymbol{\beta}_{i}$,

$$
c_{i 1} \mathbf{I}+\boldsymbol{\Gamma}_{i} \leq \widetilde{\mathbf{Q}}_{i}\left(\boldsymbol{\beta}_{i}\right)^{-1} \leq c_{i 2} \mathbf{I}+\boldsymbol{\Gamma}_{i}
$$

This assumption is valid under strictly convex penalties:
Lemma 2. Suppose that $f_{i}(\mathbf{x})$ is strictly convex in that its Hessian satisfies

$$
c_{i 1} \mathbf{I} \leq \mathcal{H}_{f_{i}}(\mathbf{x}) \leq c_{i 2} \mathbf{I}
$$

for constants $c_{i 1}, c_{i 1}>0$ and all $\mathrm{x}$. Then, the MAP and MMSE estimation functions (3) and (4) satisfy Assumption 1

Proof: See [53].

We then have the following convergence result.

Theorem 4. Consider the recursion (34) with functions $\widetilde{\mathbf{g}}_{i}(\cdot, \cdot)$ satisfying Assumption 1 and arbitrary fixed values of $\gamma_{i}>0$, for $i=1,2$. Then, from any initialization of $\boldsymbol{\beta}_{i}$, (34) converges to a unique fixed point that is invariant to the choice of $\gamma_{i}$.

Proof: See Appendix D.

\section{Convergence of the Second-Order Terms for MAP ESTIMATION}

\section{A. Convergence}

We now examine the convergence of the second-order terms $\boldsymbol{\eta}_{i}$ and $\boldsymbol{\gamma}_{i}$. The convergence results that we present here apply only to the case of MAP estimation (3) under strictly convex penalties $f_{i}(\mathbf{x})$ that satisfy the conditions in Lemma2. Furthermore, they assume that Algorithm 1 is initialized using a pair $\left(\mathbf{r}_{1}, \gamma_{1}\right)$ yielding $\mathbf{g}_{1}\left(\mathbf{r}_{1}, \gamma_{1}\right)=\widehat{\mathbf{x}}$, where $\widehat{\mathbf{x}}$ is a local minimizer of (2).

Theorem 5. Consider GEC (Algorithm 1) under the MAP estimation functions (3) with penalties $f_{i}(\mathbf{x})$ that are strictly convex functions satisfying the assumptions in Lemma 2 Construct $\mathbf{r}_{1}^{0}$ and $\gamma_{1}^{0}$ as follows:

Choose arbitrary $\gamma_{1}^{0}, \gamma_{2}^{0}>0$ and run Algorithm 1 under fixed $\gamma_{i}=\gamma_{i}^{0}$ and fixed $\boldsymbol{\eta}_{i}=\gamma_{1}^{0}+\gamma_{2}^{0}$ (for $i=1,2$ ) until convergence (as guaranteed by Theorem 4). Then record the final value of $\mathbf{r}_{1}$ as $\mathbf{r}_{1}^{0}$.

Finally, run Algorithm 1 from the initialization $\left(\mathbf{r}_{1}, \gamma_{1}\right)=$ $\left(\mathbf{r}_{1}^{0}, \gamma_{1}^{0}\right)$ without keeping $\gamma_{i}$ and $\boldsymbol{\eta}_{i}$ fixed.

(a) For all subsequent iterations, we will have $\widehat{\mathbf{x}}_{i}=\widehat{\mathbf{x}}$, where $\widehat{\mathbf{x}}$ is the unique global minimizer of (2).

(b) If $\mathbf{d}(\gamma)$ is either the vector-valued or uniform diagonalization operator, then the second-order terms $\gamma_{i}$ will converge to unique fixed points.

Proof: See Appendix E

\section{Relation to the RePlica Prediction}

Consider the separable SLR problem described in Section II-C for any matrix A and noise precision $\gamma_{w}>0$. Consider GEC under the penalty functions (15), MMSE estimation (4), and uniform diagonalization (7). Thus, $\gamma_{i}$ will have identical components of scalar value $\gamma_{i}$.

Suppose that $b_{1}(\mathbf{x})$ is the belief estimate generated at a fixed point of GEC. Since $p(\mathbf{x})$ in (14) is separable, (32) implies

$$
b_{1}\left(\mathbf{x} ; \mathbf{r}_{1}, \gamma_{1}\right) \propto \prod_{n=1}^{N} p\left(x_{n}\right) e^{-\gamma_{1}\left(x_{n}-r_{1 n}\right)^{2} / 2} .
$$


In the sequel, let $\mathbb{E}\left(\cdot \mid r_{1 n}, \gamma_{1}\right)$ and $\operatorname{var}\left(\cdot \mid r_{1 n}, \gamma_{1}\right)$ denote the mean and variance with respect to the marginal density

$$
b_{1}\left(x_{n} \mid r_{1 n}, \gamma_{1}\right) \propto p\left(x_{n}\right) e^{-\gamma_{1}\left(x_{n}-r_{1 n}\right)^{2} / 2} .
$$

From 27a , the GEC estimate $\widehat{\mathbf{x}}$ satisfies $\widehat{x}_{n}=\mathbb{E}\left(x_{n} \mid r_{1 n}, \gamma_{1}\right)$, which is the posterior mean under the estimated density (38). Also, from (27b) and the definition of the uniform diagonal operator (7), the components of $\boldsymbol{\eta}$ are identical and satisfy

$$
\eta^{-1}=\frac{1}{N} \operatorname{tr}\left(\operatorname{Cov}\left(\mathbf{x} \mid \mathbf{r}_{1}, \gamma_{1}\right)\right)=\frac{1}{N} \sum_{n=1}^{N} \operatorname{var}\left(x_{n} \mid r_{1 n}, \gamma_{1}\right)
$$

which is the average of the marginal posterior variances. Equivalently, $\eta^{-1}$ is the average estimation MSE,

$$
\eta^{-1}=\frac{1}{N} \sum_{n=1}^{N} \mathbb{E}\left[\left(x_{n}-\widehat{x}_{n}\right)^{2} \mid r_{1 n}, \gamma_{1}\right]
$$

We will show that the value for $\eta$ can be characterized in terms of the singular values of $\mathbf{A}$. Let $\mathbf{Y}:=\gamma_{w} \mathbf{A}^{\top} \mathbf{A}$, and let $S_{\mathbf{Y}}(\omega)$ denote its Stieltjes Transform (20) and $R_{\mathbf{Y}}(\omega)$ its $R$-transform (21). We then have the following.

Theorem 6. For the above problem, at any fixed point of GEC (Algorithm 11), $\eta$ and $\gamma_{1}$ satisfy the fixed-point equations

$$
\gamma_{1}=R_{\mathbf{Y}}\left(-\eta^{-1}\right), \quad \eta^{-1}=\frac{1}{N} \sum_{n=1}^{N} \operatorname{var}\left(x_{n} \mid r_{1 n}, \gamma_{1}\right),
$$

where $\operatorname{var}\left(x_{n} \mid r_{1 n}, \gamma_{1}\right)$ is the posterior variance from the density in (38).

\section{Proof: See Appendix F}

It is interesting to compare this result with that in [43], which considers exactly this estimation problem in the limit of large $N$ with certain unitarily invariant random matrices A and i.i.d. $x_{n}$. That work uses a replica symmetric (RS) analysis to predict that the asymptotic MSE $\eta^{-1}$ satisfies the fixed point equations

$$
\gamma_{1}=R_{\mathbf{Y}}\left(-\eta^{-1}\right), \quad \eta^{-1}=\mathbb{E}\left[\operatorname{var}\left(x_{n} \mid r_{1 n}, \gamma_{1}\right)\right]
$$

where the expectation is over $r_{1 n}=x_{n}+\mathcal{N}\left(0, \gamma_{1}^{-1}\right)$. This Gaussian distribution is exactly the predicted likelihood $p\left(r_{1 n} \mid x_{n}\right)$ in (33). Hence, if $x_{n}$ is i.i.d., and $r_{1 n}$ follows the likelihood in (33), then the MSE predicted from the GEC estimated posterior must satisfy the same fixed point equation as the minimum MSE predicted from the replica method in the limit as $N \rightarrow \infty$. In particular, if this equation has a unique fixed point, then the GEC-predicted MSE will match the minimum MSE as given by the replica method.

Of course, these arguments are not a rigorous proof of optimality. The analysis relies on the GEC model $p\left(\mathbf{x} \mid \mathbf{r}_{1}\right)$ with a particular choice of prior on $\mathbf{r}_{1}$. Also, the replica method itself is not rigorous. Nevertheless, the arguments do provide some hope that GEC is optimal in certain asymptotic and random regimes.

\section{RELATION TO ADMM}

We conclude by relating GEC to the well-known alternating direction method of multipliers (ADMM) [7]-[10]. Consider the MAP minimization problem (2). To solve this via ADMM, we rewrite the minimization as a constrained optimization

$$
\min _{\mathbf{x}_{1}, \mathbf{x}_{2}} f_{1}\left(\mathbf{x}_{1}\right)+f_{2}\left(\mathbf{x}_{2}\right) \text { s.t. } \mathbf{x}_{1}=\mathbf{x}_{2} \text {. }
$$

The division of the variable $\mathbf{x}$ into two variables $\mathbf{x}_{1}$ and $\mathbf{x}_{2}$ is often called variable splitting. Corresponding to the constrained optimization (42), define the augmented Lagrangian,

$$
\begin{aligned}
L_{\gamma}\left(\mathbf{x}_{1}, \mathbf{x}_{2}, \mathbf{s}\right)= & f_{1}\left(\mathbf{x}_{1}\right)+f_{2}\left(\mathbf{x}_{2}\right)+\mathbf{s}^{\top}\left(\mathbf{x}_{1}-\mathbf{x}_{2}\right) \\
& +\frac{\gamma}{2}\left\|\mathbf{x}_{1}-\mathbf{x}_{2}\right\|^{2}
\end{aligned}
$$

where $\mathbf{s}$ is a dual vector and $\gamma>0$ is an adjustable weight. The ADMM algorithm for this problem iterates the steps

$$
\begin{aligned}
\widehat{\mathbf{x}}_{1} & \leftarrow \underset{\mathbf{x}_{1}}{\arg \min } L_{\gamma}\left(\mathbf{x}_{1}, \widehat{\mathbf{x}}_{2}, \mathbf{s}\right) \\
\widehat{\mathbf{x}}_{2} & \leftarrow \underset{\mathbf{x}_{2}}{\arg \min } L_{\gamma}\left(\widehat{\mathbf{x}}_{1}, \mathbf{x}_{2}, \mathbf{s}\right) \\
\mathbf{s} & \leftarrow \mathbf{s}+\gamma\left(\widehat{\mathbf{x}}_{1}-\widehat{\mathbf{x}}_{2}\right),
\end{aligned}
$$

where it becomes evident that $\gamma$ can also be interpreted as a step size. The benefit of the ADMM method is that the minimizations involve only one penalty, $f_{1}(\mathbf{x})$ or $f_{2}(\mathbf{x})$, at a time. A classic result [7] shows that if the penalties $f_{i}(\mathbf{x})$ are convex (not necessarily smooth) and (2) has a unique minima, then the ADMM algorithm will converge to that minima. Our next result relates MAP-GEC to ADMM.

Theorem 7. Consider GEC (Algorithm 1) with the MAP estimation functions (3), but with fixed second-order terms,

$$
\gamma_{1}=\gamma_{2}=\gamma, \eta=\gamma_{1}+\gamma_{2}=2 \gamma
$$

for some fixed scalar value $\gamma>0$. Define

$$
\mathbf{s}_{i}^{k}=\gamma\left(\widehat{\mathbf{x}}_{i}^{k}-\mathbf{r}_{i}^{k}\right)
$$

Then, the outputs of GEC satisfy

$$
\begin{aligned}
\widehat{\mathbf{x}}_{1}^{k} & =\underset{\mathbf{x}_{1}}{\arg \min } L_{\gamma}\left(\mathbf{x}_{1}, \widehat{\mathbf{x}}_{2}^{k}, \mathbf{s}_{1}^{k}\right) \\
\mathbf{s}_{2}^{k} & =\mathbf{s}_{1}^{k}+\gamma\left(\widehat{\mathbf{x}}_{1}^{k}-\widehat{\mathbf{x}}_{2}^{k}\right) \\
\widehat{\mathbf{x}}_{2}^{k+1} & =\underset{\mathbf{x}_{2}}{\arg \min } L_{\gamma}\left(\widehat{\mathbf{x}}_{1}^{k}, \mathbf{x}_{2}, \mathbf{s}_{2}^{k}\right) \\
\mathbf{s}_{1}^{k+1} & =\mathbf{s}_{2}^{k}+\gamma\left(\widehat{\mathbf{x}}_{1}^{k}-\widehat{\mathbf{x}}_{2}^{k+1}\right) .
\end{aligned}
$$

Note that in the above description, we have been explicit about the iteration number $k$ to be precise about the timing of the updates. We see that a variant of ADMM can be interpreted as a special case of GEC with particular, fixed step sizes. This variant differs from the standard ADMM updates by having two updates of the dual parameters in each iteration. Alternatively, we can think of GEC as a particular variant of ADMM that uses an adaptive step size. From our discussion above, we know that the GEC algorithm can be interpreted as adapting the step-size values $\gamma^{k}$ to match the local "curvature" of the objective function. 
APPENDIX A

PROOF OF THEOREM 1

Since $\widehat{\mathbf{x}}=\widehat{\mathbf{x}}_{i}=\mathbf{g}_{i}\left(\mathbf{r}_{i}, \gamma_{i}\right)$, and $\mathbf{g}_{i}(\cdot, \cdot)$ is the MAP estimation function (3), we have

$$
\widehat{\mathbf{x}}=\underset{\mathbf{x}}{\arg \min }\left[f_{i}(\mathbf{x})+\frac{1}{2}\left\|\mathbf{x}-\mathbf{r}_{i}\right\|_{\gamma_{i}}^{2}\right] .
$$

Hence,

$$
\nabla f_{i}(\widehat{\mathbf{x}})+\gamma_{i} .\left(\widehat{\mathbf{x}}-\mathbf{r}_{i}\right)=0,
$$

where $\nabla f_{i}(\widehat{\mathbf{x}})$ denotes the gradient of $f_{i}(\mathbf{x})$ at $\mathbf{x}=\widehat{\mathbf{x}}$. Summing over $i=1,2$ and applying (18b),

$$
\nabla f_{1}(\widehat{\mathbf{x}})+\nabla f_{2}(\widehat{\mathbf{x}})=\left(\gamma_{1} \cdot \mathbf{r}_{1}+\gamma_{2} \cdot \mathbf{r}_{2}\right)-\left(\gamma_{1}+\gamma_{2}\right) \cdot \widehat{\mathbf{x}}=0,
$$

which shows that $\widehat{x}$ is a critical point of (2).

\section{APPENDIX B \\ ProOF OF THEOREM 2}

Using (11), the fixed points of line 7 of Algorithm 1 must satisfy

$$
\gamma_{j}=1 . / \mathbf{d}\left(\mathbf{Q}_{i}\right)-\gamma_{i}, \quad \mathbf{Q}_{i}=\left(\mathbf{P}_{i}+\boldsymbol{\Gamma}_{i}\right)^{-1} .
$$

Now, to prove part (a) of the theorem, suppose $\mathbf{P}_{i}=\operatorname{Diag}\left(\mathbf{p}_{i}\right)$ for some vector $\mathbf{p}_{i}$. Using (48) with the vector-valued diagonalization $\mathbf{d}(\mathbf{Q})=\operatorname{diag}(\mathbf{Q})$,

$$
\gamma_{j}=1 . / \operatorname{diag}\left[\left(\mathbf{P}_{i}+\boldsymbol{\Gamma}_{i}\right)^{-1}\right]-\gamma_{i}=\mathbf{p}_{i}+\gamma_{i}-\gamma_{i}=\mathbf{p}_{i} .
$$

Hence,

$$
\boldsymbol{\eta}=\gamma_{1}+\gamma_{2}=\mathbf{1} . / \operatorname{diag}\left[\left(\mathbf{P}_{1}+\mathbf{P}_{2}\right)^{-1}\right]=\widehat{\boldsymbol{\eta}} .
$$

In part (b) of the theorem, we use uniform diagonalization (7). Recall that $\boldsymbol{\eta}$ has identical components, which we shall call $\eta$. Likewise, $\gamma_{i}$ are vectors with identical components $\gamma_{i}$. Then from line 6 of Algorithm 1

$$
\eta^{-1}=\frac{\operatorname{tr}\left(\mathbf{Q}_{i}\right)}{N}=\frac{\operatorname{tr}\left(\left(\mathbf{P}_{i}+\gamma_{i} \mathbf{I}\right)^{-1}\right)}{N}=S_{\mathbf{P}_{i}}\left(-\gamma_{i}\right),
$$

which shows that $\gamma_{i}=-S_{\mathbf{P}_{i}}^{-1}\left(\eta^{-1}\right)$. From line 7

$$
\gamma_{j}=\eta-\gamma_{i}=\eta+S_{\mathbf{P}_{i}}^{-1}\left(\eta^{-1}\right)=R_{\mathbf{P}_{i}}\left(-\eta^{-1}\right) .
$$

Thus, using the freeness property (22),

$$
\begin{aligned}
\eta & =\gamma_{1}+\gamma_{2}=R_{\mathbf{P}_{1}}\left(-\eta^{-1}\right)+R_{\mathbf{P}_{2}}\left(-\eta^{-1}\right) \\
& =R_{\mathbf{P}_{1}+\mathbf{P}_{2}}\left(-\eta^{-1}\right)=S_{\mathbf{P}_{1}+\mathbf{P}_{2}}^{-1}\left(\eta^{-1}\right)+\eta,
\end{aligned}
$$

and hence $S_{\mathbf{P}_{1}+\mathbf{P}_{2}}(0)=\eta^{-1}$. So,

$$
\eta^{-1}=\frac{1}{N} \operatorname{tr}\left(\left(\mathbf{P}_{1}+\mathbf{P}_{2}\right)^{-1}\right)=\left[\mathbf{d}\left(\left(\mathbf{P}_{1}+\mathbf{P}_{2}\right)^{-1}\right)\right]_{1}=\widehat{\eta}^{-1} .
$$

\section{APPENDIX C}

Proof of THEOREM 3

Corresponding to the objective function (24) with moment matching constraints (27), define the Lagrangian,

$$
\begin{aligned}
& L\left(b_{1}, b_{2}, q, \boldsymbol{\beta}, \boldsymbol{\gamma}\right) \\
& :=J\left(b_{1}, b_{2}, q\right)-\sum_{i=1}^{2} \boldsymbol{\beta}_{i}^{\top}\left[\mathbb{E}\left(\mathbf{x} \mid b_{1}\right)-\mathbb{E}(\mathbf{x} \mid q)\right] \\
& \quad+\sum_{i=1}^{2} \boldsymbol{\gamma}_{i}^{\top}\left[\mathbf{d}\left(\mathbb{E}\left(\mathbf{x x}^{\top} \mid b_{1}\right)\right)-\mathbf{d}\left(\mathbb{E}\left(\mathbf{x x}^{\top} \mid q\right)\right)\right] .
\end{aligned}
$$

To show that $b_{i}$ and $q$ are stationary points of the constrained optimization, we need to show that they satisfy the moment matching constraints (27) and

$$
\begin{aligned}
b_{i} & =\underset{b_{i}}{\arg \min } L\left(b_{1}, b_{2}, q, \boldsymbol{\beta}, \boldsymbol{\gamma}\right) \\
q & =\underset{q}{\arg \max } L\left(b_{1}, b_{2}, q, \boldsymbol{\beta}, \boldsymbol{\gamma}\right)
\end{aligned}
$$

To prove (50), first observe that the Lagrangian (49) can be written as

$$
\begin{aligned}
L\left(b_{1}, b_{2}, q, \boldsymbol{\beta}, \boldsymbol{\gamma}\right)= & D\left(b_{i} \| e^{-f_{i}}\right)-\boldsymbol{\beta}_{i}^{\top} \mathbb{E}\left(\mathbf{x} \mid b_{i}\right) \\
& +\frac{1}{2} \boldsymbol{\gamma}_{i}^{\top} \mathbf{d}\left(\mathbb{E}\left(\mathbf{x} \mathbf{x}^{\top} \mid b_{i}\right)\right)+\text { const },
\end{aligned}
$$

where the constant terms do not depend on $b_{i}$. Now, for the vector-valued diagonalization operator (6), we have

$$
\gamma_{i}^{\top} \mathbf{d}\left(\mathbb{E}\left(\mathbf{x} \mathbf{x}^{\top}\right)\right)=\mathbb{E}\left[\|\mathbf{x}\|_{\gamma_{i}}^{2}\right] .
$$

The same identity also holds when $\gamma_{i}$ is a constant vector and $\mathbf{d}(\cdot)$ is the uniform diagonalization operator (7). Substituting (53) into (52), we obtain

$$
\begin{aligned}
& L\left(b_{1}, b_{2}, q, \boldsymbol{\beta}, \boldsymbol{\gamma}\right) \\
& =D\left(b_{i} \| e^{-f_{i}}\right)-\boldsymbol{\beta}_{i}^{\boldsymbol{\top}} \mathbb{E}\left(\mathbf{x} \mid b_{i}\right)+\frac{1}{2} \mathbb{E}\left[\|\mathbf{x}\|_{\gamma_{i}}^{2}\right]+\mathrm{const} \\
& \stackrel{(a)}{=} D\left(b_{i} \| e^{-f_{i}}\right)+\frac{1}{2} \mathbb{E}\left[\left\|\mathbf{x}-\mathbf{r}_{i}\right\|_{\gamma_{i}}^{2}\right]+\text { const } \\
& \stackrel{(b)}{=}-H\left(b_{i}\right)+\mathbb{E}\left[f_{i}(\mathbf{x})+\frac{1}{2}\left\|\mathbf{x}-\mathbf{r}_{i}\right\|_{\gamma_{i}}^{2}\right]+\text { const } \\
& \stackrel{(c)}{=} D\left(b_{i} \| p_{i}\left(\cdot \mid \mathbf{r}_{i}, \gamma_{i}\right)\right)+\text { const, }
\end{aligned}
$$

where in step (a) we used the fact that $\boldsymbol{\beta}_{i}=\gamma_{i} \cdot \mathbf{r}_{i}$, and in steps (b) and (c) we used the definitions of KL divergence and $p_{i}(\cdot)$ from (5). Thus, the minimization in (50) yields (28).

The maximization over $q$ in (51) is computed similarly. Removing the terms that do not depend on $q$,

$$
\begin{aligned}
& L\left(b_{1}, b_{2}, q, \boldsymbol{\beta}, \boldsymbol{\gamma}\right) \\
& =H(q)+\sum_{i=1}^{2} \boldsymbol{\beta}_{i}^{\top} \mathbb{E}(\mathbf{x} \mid q)-\frac{1}{2} \sum_{i=1}^{2} \mathbb{E}\left[\|\mathbf{x}\|_{\boldsymbol{\gamma}_{i}}^{2}\right]+\mathrm{const} \\
& \stackrel{(a)}{=} H(q)+(\boldsymbol{\eta} \cdot \widehat{\mathbf{x}})^{\top} \mathbb{E}(\mathbf{x} \mid q)-\frac{1}{2} \mathbb{E}\left[\|\mathbf{x}\|_{\boldsymbol{\eta}}^{2}\right]+\mathrm{const} \\
& \stackrel{(b)}{=} H(q)-\frac{1}{2} \mathbb{E}\left[\|\mathbf{x}-\widehat{\mathbf{x}}\|_{\boldsymbol{\eta}}^{2}\right]+\mathrm{const} \\
& \stackrel{(c)}{=}-D(q \| \widehat{q})+\text { const, }
\end{aligned}
$$


where step (a) uses the facts that $\gamma_{1}+\gamma_{2}=\boldsymbol{\eta}$ and

$$
\boldsymbol{\beta}_{1}+\boldsymbol{\beta}_{2}=\gamma_{1} \cdot \mathbf{r}_{1}+\boldsymbol{\gamma}_{2} \cdot \mathbf{r}_{2}=\boldsymbol{\eta} \cdot \widehat{\mathbf{x}},
$$

step (b) follows by completing the square, and step (c) uses the density

$$
\widehat{q}(\mathbf{x}) \propto \exp \left[\frac{1}{2}\|\mathbf{x}-\widehat{\mathbf{x}}\|_{\boldsymbol{\eta}}^{2}\right] .
$$

Hence, the maximum in (51) is given by $q=\widehat{q}$, which matches (29).

Also, from lines 4 and 6 of Algorithm 1 and (4) and (12),

$$
\widehat{\mathbf{x}}=\mathbb{E}\left(\mathbf{x} \mid b_{i}\right), \quad \mathbf{1} \cdot / \boldsymbol{\eta}=\mathbf{d}\left(\operatorname{Cov}\left(\mathbf{x} \mid b_{i}\right)\right) .
$$

Since $\widehat{q}$ is Gaussian, its mean and covariance matrix are

$$
\mathbb{E}(\mathbf{x} \mid q)=\widehat{\mathbf{x}}, \quad \operatorname{Cov}(\mathbf{x} \mid q)=\operatorname{Diag}(\mathbf{1} . / \boldsymbol{\eta}) .
$$

This proves that the densities satisfy the moment matching constraints (27).

\section{APPENDIX D \\ Proof of Theorem 4}

Let $\gamma=\gamma_{1}+\gamma_{2}$ and $\boldsymbol{\Gamma}=\operatorname{Diag}(\gamma)$. Define the scaled variables $\mathbf{v}_{i}=\boldsymbol{\Gamma}^{-1 / 2} \boldsymbol{\beta}_{i}$. Since $\boldsymbol{\gamma}>0$, it suffices to prove the convergence of $\mathbf{v}_{i}$. We can rewrite the update (34) as

$$
\mathbf{v}_{j} \leftarrow F_{i}\left(\mathbf{v}_{i}\right):=\boldsymbol{\Gamma}^{1 / 2} \widetilde{\mathbf{g}}_{i}\left(\boldsymbol{\Gamma}^{1 / 2} \mathbf{v}_{i}, \gamma_{i}\right)-\mathbf{v}_{i} .
$$

So, we have that the updates are given by the recursion

$$
\mathbf{v}_{2}=F_{1}\left(\mathbf{v}_{2}\right)=F_{1}\left(F_{2}\left(\mathbf{v}_{2}\right)\right) .
$$

If $\mathbf{J}_{i}\left(\mathbf{v}_{i}\right)=\partial F_{i}\left(\mathbf{v}_{i}\right) / \partial \mathbf{v}_{i}$ is the Jacobian of transformation, then, by the chain rule, the Jacobian of the composition is $\partial\left(F_{1} \circ F_{2}\right) / \partial \mathbf{v}_{1}=\mathbf{J}_{1} \mathbf{J}_{2}$. A standard contraction mapping result [54] shows that if, for some $\rho<1$,

$$
\left\|\mathbf{J}_{1}\left(\mathbf{v}_{1}\right) \mathbf{J}_{2}\left(\mathbf{v}_{2}\right)\right\| \leq \rho,
$$

then $\mathbf{v}_{i}$ converges linearly to a unique fixed point.

So, we need to characterize the norms of the Jacobians. First, the Jacobian of the update function in (57) is

$$
\mathbf{J}_{i}\left(\mathbf{v}_{i}\right)=\boldsymbol{\Gamma}^{1 / 2} \widetilde{\mathbf{Q}}_{i} \boldsymbol{\Gamma}^{1 / 2}-\mathbf{I}, \quad \widetilde{\mathbf{Q}}_{i}=\frac{\partial \widetilde{\mathbf{g}}_{i}\left(\boldsymbol{\beta}_{i}, \boldsymbol{\gamma}_{i}\right)}{\partial \boldsymbol{\beta}_{i}}
$$

Using Assumption 1, $\widetilde{\mathbf{Q}}_{i}$ is symmetric and hence so is $\mathbf{J}_{i}$. Also, using (36) and the fact that $\boldsymbol{\eta}=\gamma_{1}+\gamma_{2}$,

$$
\begin{aligned}
\mathbf{J}_{i}\left(\mathbf{v}_{i}\right) & \leq \operatorname{Diag}\left[\left(\gamma_{1}+\gamma_{2}\right) \cdot /\left(c_{i 2} \mathbf{1}+\gamma_{i}\right)-\mathbf{1}\right] \\
& =\operatorname{Diag}\left[\left(\gamma_{j}-c_{i 2} \mathbf{1}\right) \cdot /\left(\boldsymbol{\gamma}_{i}+c_{i 2} \mathbf{1}\right)\right],
\end{aligned}
$$

for $(i, j)=(1,2)$ or $(2,1)$. Similarly,

$$
\begin{aligned}
\mathbf{J}_{i}\left(\mathbf{v}_{i}\right) & \geq \operatorname{Diag}\left[\left(\boldsymbol{\gamma}_{1}+\boldsymbol{\gamma}_{2}\right) \cdot /\left(c_{i 1} \mathbf{1}+\boldsymbol{\gamma}_{i}\right)-\mathbf{1}\right] \\
& =\operatorname{Diag}\left[\left(\boldsymbol{\gamma}_{j}-c_{i 1} \mathbf{1}\right) \cdot /\left(\boldsymbol{\gamma}_{i}+c_{i 1} \mathbf{1}\right)\right] .
\end{aligned}
$$

Thus, the matrix absolute value of $\mathbf{J}_{i}$ (i.e. from the spectral theorem, not componentwise) satisfies

$$
\left|\mathbf{J}_{i}\right| \leq \operatorname{Diag}\left[\frac{\left|\gamma_{j}-\mathbf{q}_{i}\right|}{\left|\gamma_{i}+\mathbf{q}_{i}\right|}\right],
$$

where $\mathbf{q}_{i}$ has components $q_{i n}=c_{i 1}$ or $c_{i 2}$. Thus,

$$
\left|\mathbf{J}_{1}\right|\left|\mathbf{J}_{2}\right| \leq \operatorname{Diag}\left[\frac{\left|\gamma_{2}-\mathbf{q}_{1}\right|\left|\gamma_{1}-\mathbf{q}_{2}\right|}{\left|\gamma_{1}+\mathbf{q}_{1}\right|\left|\gamma_{2}+\mathbf{q}_{2}\right|}\right] .
$$

Now for each component $n$,

$$
\left|\gamma_{2 n}-q_{1 n}\right|\left|\gamma_{1 n}-q_{2 n}\right|<\left|\gamma_{1 n}+q_{1 n}\right|\left|\gamma_{2 n}+q_{2 n}\right|,
$$

since all the terms are positive. Since this is true for all $n$, there must exist a $\rho \in[0,1)$ such that,

$$
\left|\mathbf{J}_{1}\right|\left|\mathbf{J}_{2}\right| \leq \rho \mathbf{I}
$$

Note that the value of $\rho$ can be selected independently of $\mathbf{v}_{i}$. Therefore, the norm of the Jacobian product satisfies

$$
\left\|\mathbf{J}_{1} \mathbf{J}_{2}\right\| \leq\left\|\left|\mathbf{J}_{1}\right|\left|\mathbf{J}_{2}\right|\right\| \leq \rho
$$

for all $\mathbf{v}_{i}$. Hence, the mapping is a contraction.

\section{APPENDIX E \\ Proof of ThEOREM 5}

We start by proving part (a). First, recall that $\left(\mathbf{r}_{1}^{0}, \gamma_{1}^{0}\right)$ were constructed by running Algorithm 1 to convergence under fixed $\gamma_{i}^{0}>0$ and $\eta_{i}^{0}=\gamma_{1}^{0}+\gamma_{2}^{0}$ for $i=1,2$. Theorem 1 studied this recursion and showed that it yields final values " $\mathbf{r}_{i}^{0}$ " of $\mathbf{r}_{i}$ for which the corresponding estimates $\widehat{\mathbf{x}}_{i}^{0}:=\mathrm{g}_{i}\left(\mathbf{r}_{i}^{0}, \gamma_{i}^{0}\right)$ satisfy $\widehat{\mathbf{x}}_{1}^{0}=\widehat{\mathbf{x}}_{2}^{0}=\widehat{\mathbf{x}}$, where $\widehat{\mathbf{x}}$ is a local minima of (2). Since we have assumed that $f_{i}(\mathbf{x})$ are strictly convex, $\widehat{\mathbf{x}}$ is the unique global minimizer. Theorem 5 then considers what happens when Algorithm 1 is run from the initialization $\left(\mathbf{r}_{1}^{0}, \gamma_{1}^{0}\right)$ without holding $\boldsymbol{\gamma}_{i}, \boldsymbol{\eta}_{i}$ fixed.

We now show that $\widehat{\mathbf{x}}_{1}=\widehat{\mathbf{x}}_{2}=\widehat{\mathbf{x}}$ for all iterations. We prove this by induction. Suppose that $\widehat{\mathbf{x}}_{i}=\widehat{\mathbf{x}}$ (which we know holds for $i=1$ during the first iteration due to the construction of the initialization $\left.\widehat{\mathbf{x}}_{1}^{0}\right)$. Since $\widehat{\mathbf{x}}_{i}=\mathbf{g}_{i}\left(\mathbf{r}_{i}, \gamma_{i}\right)$ with $\mathbf{g}_{i}(\cdot, \cdot)$ being the minimizer in (3), $\widehat{\mathbf{x}}=\widehat{\mathbf{x}}_{i}$ must satisfy the first-order condition

$$
\nabla f_{i}(\widehat{\mathbf{x}})+\gamma_{i} .\left(\widehat{\mathbf{x}}-\mathbf{r}_{i}\right)=0 .
$$

Therefore,

$$
\begin{aligned}
\nabla f_{j}(\widehat{\mathbf{x}})+\gamma_{j} \cdot\left(\widehat{\mathbf{x}}-\mathbf{r}_{j}\right) \\
\quad \stackrel{(a)}{=}-\nabla f_{i}(\widehat{\mathbf{x}})+\gamma_{j} \cdot\left(\widehat{\mathbf{x}}-\mathbf{r}_{j}\right) \\
\quad \stackrel{(b)}{=}-\nabla f_{i}(\widehat{\mathbf{x}})+\left(\boldsymbol{\eta}_{i}-\gamma_{i}\right) \cdot \widehat{\mathbf{x}}-\gamma_{j} \cdot \mathbf{r}_{j} \\
\quad \stackrel{(c)}{=}-\gamma_{i} \cdot \mathbf{r}_{i}+\boldsymbol{\eta}_{i} \cdot \widehat{\mathbf{x}}-\gamma_{j} \cdot \mathbf{r}_{j} \stackrel{(d)}{=} 0,
\end{aligned}
$$

where (a) follows from the fact that $\widehat{\mathbf{x}}$ is the minimizer of (2) and so $\nabla f_{i}(\widehat{\mathbf{x}})+\nabla f_{j}(\widehat{\mathbf{x}})=0$; (b) follows from line 7 of Algorithm 1, (c) follows from the induction hypothesis [63); and (d) follows from line 8 . Hence $\widehat{\mathbf{x}}$ satisfies the first-order minimization conditions for $\widehat{\mathbf{x}}_{j}=\mathbf{g}_{j}\left(\mathbf{r}_{j}, \gamma_{j}\right)$, so $\widehat{\mathbf{x}}_{j}=\widehat{\mathbf{x}}$. This proves part (a).

We now turn to part (b). We will prove this part for the vector-valued diagonalization operator (6). The proof for the uniform diagonalization operator (7) is similar, but easier. Now, from part (a), we can assume that $\widehat{\mathbf{x}}_{i}=\widehat{\mathbf{x}}$ for all iterations. From (11), we see that $\mathbf{Q}_{i}$ in line 5 is given by

$$
\mathbf{Q}_{i}=\left(\mathbf{P}_{i}+\boldsymbol{\Gamma}_{i}\right)^{-1}, \quad \mathbf{P}_{i}=\mathcal{H}_{f_{i}}(\widehat{\mathbf{x}}),
$$


where $\mathcal{H}_{f_{i}}(\widehat{\mathbf{x}})$ is the Hessian. Hence, from line 7, the updates of the second-order terms are given by

$$
\gamma_{j} \leftarrow G^{i}\left(\gamma_{i}\right):=1 . / \operatorname{diag}\left(\left(\mathbf{P}_{i}+\boldsymbol{\Gamma}_{i}\right)^{-1}\right)-\gamma_{i} .
$$

Note that, since the penalty functions $f_{i}(\mathbf{x})$ satisfy the assumptions in Lemma 2, we have $\mathbf{P}_{i}:=\mathcal{H}_{f_{i}}(\widehat{\mathbf{x}})>0$. Also, we can write the update on $\gamma_{1}$ as

$$
\gamma_{1} \leftarrow G^{2} \circ G^{1}\left(\gamma_{1}\right)
$$

where $G^{2} \circ G^{1}$ is the composition map.

Now, to analyze this update, consider a general map of the form

$$
G(\gamma):=1 . / \operatorname{diag}\left((\mathbf{P}+\boldsymbol{\Gamma})^{-1}\right)-\gamma,
$$

where, as before, we use the notation $\boldsymbol{\Gamma}=\operatorname{diag}(\gamma)$ and $\mathbf{P}>0$. We prove four properties of a map of this form: For any $\gamma>0$,

(i) Non-negative: $G(\gamma) \geq 0$;

(ii) Non-decreasing: $G\left(\gamma^{\prime}\right) \geq G(\gamma)$ when $\gamma^{\prime}>\gamma$;

(iii) Sub-multiplicative: For any $\alpha>1, G(\alpha \gamma) \leq \alpha G(\gamma)$;

(iv) Bounded: There exists a $M$ such that $G(\gamma) \leq M$.

If we can show that $G(\gamma)$ in (67) satisfies these four properties, then so does $G^{i}\left(\gamma_{i}\right)$ in (65) and hence their composition $G^{1} \circ G^{2}$. It is then shown in [55] that the update in (66) must converge to a unique fixed point. So, we must simply prove that $G(\gamma)$ in (67) satisfies properties (i) to (iv).

For property (i), observe that the components of $G(\gamma)$ are given by

$$
G_{i}(\gamma)=\left[(\mathbf{P}+\boldsymbol{\Gamma})^{-1}\right]_{i i}^{-1}-\gamma_{i} .
$$

Since $\mathbf{P} \geq 0$,

$$
G_{i}(\boldsymbol{\gamma}) \geq\left[\boldsymbol{\Gamma}^{-1}\right]_{i i}^{-1}-\gamma_{i}=0 .
$$

So $G_{i}(\gamma) \geq 0$. This proves property (i). Next, we prove that it is increasing. Let

$$
\mathbf{S}=(\mathbf{P}+\boldsymbol{\Gamma})^{-1},
$$

so that we can write $G_{i}(\gamma)$ as

$$
G_{i}(\gamma)=S_{i i}^{-1}-\gamma_{i}
$$

Then

$$
\begin{aligned}
& \frac{\partial G_{i}(\gamma)}{\partial \gamma_{j}}=-S_{i i}^{-2} \frac{\partial}{\partial \gamma_{j}} \mathbf{e}_{i}^{\top} \mathbf{S e}_{i}-\delta_{i-j} \\
& =-S_{i i}^{-2} \mathbf{e}_{i}^{\top}\left[\frac{\partial}{\partial \gamma_{j}}(\mathbf{P}+\boldsymbol{\Gamma})^{-1}\right] \mathbf{e}_{i}-\delta_{i-j} \\
& =S_{i i}^{-2} \mathbf{e}_{i}^{\top}(\mathbf{P}+\boldsymbol{\Gamma})^{-1}\left[\frac{\partial}{\partial \gamma_{j}}(\mathbf{P}+\boldsymbol{\Gamma})\right](\mathbf{P}+\boldsymbol{\Gamma})^{-1} \mathbf{e}_{i}-\delta_{i-j} \\
& =S_{i i}^{-2} \mathbf{e}_{i}^{\top} \mathbf{S}\left[\mathbf{e}_{j} \mathbf{e}_{j}^{\top}\right] \mathbf{S e}_{i}-\delta_{i-j} \\
& =S_{i j}^{2} / S_{i i}^{2}-\delta_{i-j} .
\end{aligned}
$$

For $i \neq j$, we see that

$$
\frac{\partial G_{i}(\gamma)}{\partial \gamma_{j}}=\frac{S_{i j}^{2}}{S_{i i}^{2}} \geq 0
$$

and for $i=j$, we have

$$
\frac{\partial G_{i}(\gamma)}{\partial \gamma_{j}}=\frac{S_{i i}^{2}}{S_{i i}^{2}}-1=0 .
$$

Hence, the function is non-decreasing, which proves property (ii). Next, we need to show that is sub-multiplicative. Suppose $\alpha>1$. Then

$$
\begin{aligned}
G(\alpha \boldsymbol{\gamma}) & =\mathbf{1} . / \operatorname{diag}\left((\mathbf{P}+\alpha \boldsymbol{\Gamma})^{-1}\right)-\alpha \boldsymbol{\gamma} \\
& \leq \mathbf{1} . / \operatorname{diag}\left((\alpha \mathbf{Q}+\alpha \boldsymbol{\Gamma})^{-1}\right)-\alpha \boldsymbol{\gamma}=\alpha G(\boldsymbol{\gamma}),
\end{aligned}
$$

which proves property (iii). Lastly, we need to show it is bounded above. First notice that we can write

$$
\begin{aligned}
G(\gamma) & =\operatorname{diag}\left(\mathbf{I}-\boldsymbol{\Gamma}(\mathbf{P}+\boldsymbol{\Gamma})^{-1}\right) \cdot / \operatorname{diag}\left((\mathbf{P}+\boldsymbol{\Gamma})^{-1}\right) \\
& =\operatorname{diag}\left(\mathbf{P}(\mathbf{P}+\boldsymbol{\Gamma})^{-1}\right) \cdot / \operatorname{diag}\left((\mathbf{P}+\boldsymbol{\Gamma})^{-1}\right) \\
& =\operatorname{diag}\left(\mathbf{P}(\mathbf{P}+\boldsymbol{\Gamma})^{-1} \boldsymbol{\Gamma}\right) \cdot / \operatorname{diag}\left((\mathbf{P}+\boldsymbol{\Gamma})^{-1} \boldsymbol{\Gamma}\right) \\
& =\operatorname{diag}\left(\left(\boldsymbol{\Gamma}^{-1}+\mathbf{P}^{-1}\right)^{-1}\right) \cdot / \operatorname{diag}\left(\left(\boldsymbol{\Gamma}^{-1} \mathbf{P}+\mathbf{I}\right)^{-1}\right) .
\end{aligned}
$$

Then as $\gamma \rightarrow \infty$, we have

$$
G(\boldsymbol{\gamma}) \rightarrow \operatorname{diag}\left(\left(\mathbf{P}^{-1}\right)^{-1}\right) \cdot / \operatorname{diag}\left(\mathbf{I}^{-1}\right)=\operatorname{diag}(\mathbf{P}) .
$$

If $\mathbf{P}$ is the Hessian of a penalty function that satisfies the assumptions in Lemma 2, then $\operatorname{diag}(\mathbf{P})$ is be bounded from above, implying that $G(\gamma)$ is also bounded from above. This proves properties (i) to (iv) above.

\section{APPENDIX F}

\section{Proof of THEOREM 6}

For the penalty $f_{2}(\mathbf{x})$ in $(15)$, the belief estimate $b_{2}(\mathbf{x})$ in (28) is Gaussian with covariance matrix

$$
\operatorname{Cov}\left(\mathbf{x} \mid b_{2}\right)=\left[\gamma_{w} \mathbf{A}^{*} \mathbf{A}+\gamma_{2} \mathbf{I}\right]^{-1}=\left[\mathbf{Y}+\gamma_{2} \mathbf{I}\right]^{-1},
$$

where $\mathbf{Y}=\gamma_{w} \mathbf{A}^{\top} \mathbf{A}$. From (11) and line 6 in Algorithm 1 , $\mathbf{Q}_{2}=\operatorname{Cov}\left(\mathbf{x} \mid b_{2}\right)$. Under the uniform diagonalization operator (7), $\boldsymbol{\eta}$ has identical components $\eta$ such that

$$
\eta^{-1}=\frac{1}{N} \operatorname{tr}\left(\mathbf{Q}_{2}\right)=S_{\mathbf{Y}}\left(-\gamma_{2}\right),
$$

where $S_{\mathbf{Y}}(\omega)$ is the Stieltjes transform (20). Hence,

$$
\gamma_{2}=-S_{\mathbf{Y}}^{-1}\left(\eta^{-1}\right) .
$$

Also, $\gamma_{i}$ has identical components $\gamma_{i}$ for each $i=1,2$. Thus from line 7 of Algorithm 1

$$
\gamma_{1}=\eta-\gamma_{2}=\eta+S_{\mathbf{Y}}^{-1}\left(\eta^{-1}\right)=R_{\mathbf{Y}}\left(-\eta^{-1}\right) .
$$

This proves the first equation in (40). The second equation is exactly (39).

\section{APPENDIX G \\ Proof OF THEOREM 7}

To prove 47a ,

$$
\begin{aligned}
\widehat{\mathbf{x}}_{1}^{k} & \stackrel{(a)}{=} \underset{\mathbf{x}_{1}}{\arg \min } f_{1}\left(\mathbf{x}_{1}\right)+\frac{\gamma}{2}\left\|\mathbf{x}_{1}-\mathbf{r}_{1}^{k}\right\|^{2} \\
& \stackrel{(b)}{=} \underset{\mathbf{x}_{1}}{\arg \min } f_{1}\left(\mathbf{x}_{1}\right)+\frac{\gamma}{2}\left\|\mathbf{x}_{1}-\widehat{\mathbf{x}}_{2}^{k}-\gamma^{-1} \mathbf{s}_{1}^{k}\right\|^{2} \\
& \stackrel{(c)}{=} \underset{\mathbf{x}_{1}}{\arg \min } f_{1}\left(\mathbf{x}_{1}\right)+\frac{\gamma}{2}\left\|\mathbf{x}_{1}-\widehat{\mathbf{x}}_{2}^{k}\right\|^{2}+\left(\mathbf{s}_{1}^{k}\right)^{\top} \mathbf{x}_{1} \\
& \stackrel{(d)}{=} \underset{\mathbf{x}_{1}}{\arg \min } L\left(\mathbf{x}_{1}, \widehat{\mathbf{x}}_{2}, \mathbf{s}_{1}^{k}\right),
\end{aligned}
$$


where (a) follows from $\widehat{\mathbf{x}}_{1}^{k}=\mathbf{g}_{1}\left(\mathbf{r}_{1}^{k}, \gamma\right)$ and the definition of the MAP estimation function in (3); (b) follows from the definition of $\mathbf{s}_{1}^{k}$ in (46); (c) follows by expanding the squares and (d) follows from the augmented Lagrangian in (43). The proof of 47c is similar.

To prove 47d), first observe from the update of $\mathbf{r}_{j}$ in line (8) of Algorithm 1 and the fact that $\eta=2 \gamma$, we have

$$
\gamma \mathbf{r}_{2}^{k}=2 \gamma \widehat{\mathbf{x}}_{1}^{k}-\gamma \mathbf{r}_{1}^{k}
$$

Therefore,

$$
\begin{aligned}
\mathbf{s}_{2}^{k} \stackrel{(a)}{=} & \gamma\left(\mathbf{r}_{2}^{k}-\widehat{\mathbf{x}}_{1}^{k}\right) \\
& \stackrel{(b)}{=} \gamma\left(\widehat{\mathbf{x}}_{1}^{k}-\mathbf{r}_{1}^{k}\right)=\mathbf{s}_{1}^{k}+\gamma\left(\widehat{\mathbf{x}}_{1}^{k}-\widehat{\mathbf{x}}_{2}^{k}\right),
\end{aligned}
$$

where (a) follows from the definition of $\mathbf{s}_{2}^{k}$ in 46); (b) follows from (69) and (c) follows from (46). The proof of (47b) is similar.

\section{REFERENCES}

[1] A. Chambolle, R. A. DeVore, N. Y. Lee, and B. J. Lucier, "Nonlinear wavelet image processing: Variational problems, compression, and noise removal through wavelet shrinkage," IEEE Trans. Image Process., vol. 7, no. 3, pp. 319-335, Mar. 1998.

[2] I. Daubechies, M. Defrise, and C. D. Mol, "An iterative thresholding algorithm for linear inverse problems with a sparsity constraint," Commun. Pure Appl. Math., vol. 57, no. 11, pp. 1413-1457, Nov. 2004.

[3] S. J. Wright, R. D. Nowak, and M. Figueiredo, "Sparse reconstruction by separable approximation," IEEE Trans. Signal Process., vol. 57, no. 7, pp. 2479-2493, Jul. 2009.

[4] A. Beck and M. Teboulle, "A fast iterative shrinkage-thresholding algorithm for linear inverse problem," SIAM J. Imag. Sci., vol. 2, no. 1, pp. 183-202, 2009.

[5] Y. E. Nesterov, "Gradient methods for minimizing composite objective function," CORE Report, 2007.

[6] J. Bioucas-Dias and M. Figueiredo, "A new TwIST: Two-step iterative shrinkage/thresholding algorithms for image restoration," IEEE Trans. Image Process., vol. 16, no. 12, pp. 2992 - 3004, Dec. 2007.

[7] S. Boyd, N. Parikh, E. Chu, B. Peleato, and J. Eckstein, "Distributed optimization and statistical learning via the alternating direction method of multipliers," Found. Trends Mach. Learn., vol. 3, pp. 1-122, 2010.

[8] E. Esser, X. Zhang, and T. F. Chan, "A general framework for a class of first order primal-dual algorithms for convex optimization in imaging science," SIAM J. Imaging Sci., vol. 3, no. 4, pp. 1015-1046, 2010.

[9] A. Chambolle and T. Pock, "A first-order primal-dual algorithm for convex problems with applications to imaging," J. Math. Imaging Vis., vol. 40, pp. 120-145, 2011

[10] B. He and X. Yuan, "Convergence analysis of primal-dual algorithms for a saddle-point problem: From contraction perspective," SIAM J. Imaging Sci., vol. 5, no. 1, pp. 119-149, 2012.

[11] M. Pereyra, P. Schniter, E. Chouzenoux, J.-C. Pesquet, J.-Y. Tourneret, A. Hero, and S. McLaughlin, "A survey of stochastic simulation and optimization methods in signal processing," IEEE J. Sel. Topics Signal Process., to appear 2016 (see also arXiv:1505.00273).

[12] M. W. Seeger and H. Nickisch, "Fast convergent algorithms for expectation propagation approximate bayesian inference," in International Conference on Artificial Intelligence and Statistics, 2011, pp. 652-660.

[13] S. Rangan, A. K. Fletcher, P. Schniter, and U. S. Kamilov, "Inference for generalized linear models via alternating directions and Bethe free energy minimization," in Proc. IEEE ISIT, 2015, pp. 1640 - 1644.

[14] J. Pearl, Probabilistic Reasoning in Intelligent Systems: Networks of Plausible Inference. San Mateo, CA: Morgan Kaufmann Publ., 1988.

[15] J. S. Yedidia, W. T. Freeman, and Y. Weiss, "Understanding belief propagation and its generalizations," in Exploring Artificial Intelligence in the New Millennium. San Francisco, CA: Morgan Kaufmann Publishers, 2003, pp. 239-269.

[16] T. P. Minka, "A family of algorithms for approximate Bayesian inference," Ph.D. dissertation, Massachusetts Institute of Technology, Cambridge, MA, 2001.

[17] M. Opper and O. Winther, "Expectation consistent free energies for approximate inference," in Proc. NIPS, 2004, pp. 1001-1008.
[18] M. Seeger, "Expectation propagation for exponential families," $E P F L$ REPORT-161464, 2005.

[19] D. L. Donoho, A. Maleki, and A. Montanari, "Message-passing algorithms for compressed sensing," Proc. Nat. Acad. Sci., vol. 106, no. 45, pp. 18914-18919, Nov. 2009.

[20] S. Rangan, "Generalized approximate message passing for estimation with random linear mixing," in Proc. IEEE Int. Symp. Inform. Theory, Saint Petersburg, Russia, Jul.-Aug. 2011, pp. 2174-2178.

[21] D. L. Donoho, A. Maleki, and A. Montanari, "Message passing algorithms for compressed sensing I: motivation and construction," in Proc. Info. Theory Workshop, Jan. 2010.

[22] — "Message passing algorithms for compressed sensing II: analysis and validation," in Proc. Info. Theory Workshop, Jan. 2010.

[23] S. Rangan, "Estimation with random linear mixing, belief propagation and compressed sensing," in Proc. Conf. on Inform. Sci. \& Sys., Princeton, NJ, Mar. 2010, pp. 1-6.

[24] M. Bayati and A. Montanari, "The dynamics of message passing on dense graphs, with applications to compressed sensing," IEEE Trans. Inform. Theory, vol. 57, no. 2, pp. 764-785, Feb. 2011.

[25] A. Javanmard and A. Montanari, "State evolution for general approximate message passing algorithms, with applications to spatial coupling," arXiv:1211.5164 [math.PR]., Nov. 2012.

[26] A. K. Fletcher and S. Rangan, "Scalable inference for neuronal connectivity from calcium imaging," in Proc. Neural Information Processing Systems, 2014, pp. 2843-2851.

[27] A. K. Fletcher, S. Rangan, L. Varshney, and A. Bhargava, "Neural reconstruction with approximate message passing (NeuRAMP)," in Proc. Neural Information Process. Syst., Granada, Spain, Dec. 2011.

[28] P. Schniter, "A message-passing receiver for BICM-OFDM over unknown clustered-sparse channels," vol. 5, no. 8, pp. 1462-1474, Dec. 2011.

[29] S. Som and P. Schniter, "Compressive imaging using approximate message passing and a Markov-tree prior," IEEE Trans. Signal Process. vol. 60, no. 7, pp. 3439-3448, Jul. 2012.

[30] J. Parker, P. Schniter, and V. Cevher, "Bilinear generalized approximate message passing -Part II: Applications," IEEE Trans. Signal Processing, vol. 62, no. 22, pp. 5854-5867, 2013.

[31] P. Schniter and S. Rangan, "Compressive phase retrieval via generalized approximate message passing," IEEE Trans. Signal Process., vol. 63, no. 4, pp. 1043-1055, 2015.

[32] J. Vila, P. Schniter, and J. Meola, "Hyperspectral image unmixing via turbo bilinear approximate message passing," IEEE Trans. Comp. Imag., vol. 1, no. 3, pp. 143-158, 2015.

[33] J. Ziniel, P. Schniter, and P. Sederberg, "Binary linear classification and feature selection via generalized approximate message passing," IEEE Trans. Signal Process., vol. 63, no. 8, pp. 2020-2032, 2015.

[34] M. Opper and O. Winther, "Gaussian processes for classification: Meanfield algorithms," Neural Computation, vol. 12, no. 11, pp. 2655-2684, 2000.

[35] - "Adaptive and self-averaging Thouless-Anderson-Palmer meanfield theory for probabilistic modeling," Physical Review E, vol. 64, no. 5 , p. $056131,2001$.

[36] S. Rangan, P. Schniter, and A. Fletcher, "On the convergence of approximate message passing with arbitrary matrices," in Proc. ISIT, Jul. 2014, pp. 236-240.

[37] F. Caltagirone, L. Zdeborová, and F. Krzakala, "On convergence of approximate message passing," in Proc. ISIT, Jul. 2014, pp. 1812-1816.

[38] F. Krzakala, A. Manoel, E. W. Tramel, and L. Zdeborová, "Variational free energies for compressed sensing," in Proc. ISIT, Jul. 2014, pp. $1499-1503$

[39] M. Seeger, "Bayesian inference and optimal design for the sparse linear model," J. Machine Learning Research, vol. 9, pp. 759-813, Sep. 2008.

[40] J. Vila, P. Schniter, S. Rangan, F. Krzakala, and L. Zdeborová, "Adaptive damping and mean removal for the generalized approximate message passing algorithm," in Proc. IEEE ICASSP, 2015, to appear.

[41] A. Manoel, F. Krzakala, E. W. Tramel, and L. Zdeborová, "Sparse estimation with the swept approximated message-passing algorithm," arXiv:1406.4311, Jun. 2014.

[42] H. Nickisch and M. W. Seeger, "Convex variational Bayesian inference for large scale generalized linear models," in Proc. ICML, 2009, pp. $761-768$.

[43] A. M. Tulino, G. Caire, S. Verdu, and S. Shamai, "Support recovery with sparsely sampled free random matrices," IEEE Transactions on Information Theory, vol. 59, no. 7, pp. 4243-4271, 2013.

[44] T. Tanaka and M. Okada, "Approximate belief propagation, density evolution, and neurodynamics for CDMA multiuser detection," IEEE Trans. Inform. Theory, vol. 51, no. 2, pp. 700-706, Feb. 2005. 
[45] D. Guo and S. Verdú, "Randomly spread CDMA: Asymptotics via statistical physics," IEEE Trans. Inform. Theory, vol. 51, no. 6, pp. 1983-2010, Jun. 2005.

[46] S. Rangan, A. Fletcher, and V. K. Goyal, "Asymptotic analysis of MAP estimation via the replica method and applications to compressed sensing," IEEE Trans. Inform. Theory, vol. 58, no. 3, pp. 1902-1923, Mar. 2012.

[47] M. Vehkapera, Y. Kabashima, and S. Chatterjee, "Analysis of regularized ls reconstruction and random matrix ensembles in compressed sensing," in Proc. IEEE ISIT, 2014, pp. 3185-3189.

[48] B. Çakmak, O. Winther, and B. H. Fleury, "S-AMP: Approximate message passing for general matrix ensembles," in Proc. IEEE Information Theory Workshop (ITW), 2014, pp. 192-196.

[49] —_, "S-AMP for non-linear observation models," 2015.

[50] J. A. Nelder and R. W. M. Wedderburn, "Generalized linear models," $J$. Royal Stat. Soc. Series A, vol. 135, pp. 370-385, 1972.

[51] P. McCullagh and J. A. Nelder, Generalized linear models, 2nd ed. Chapman \& Hall, 1989.

[52] A. Tulino and S. Verdú, "Random matrix theory and wireless communications," Found. Trends Commun. Info. Thy., vol. 1, pp. 1-182, 2004

[53] S. Rangan, A. K. Fletcher, P. Schniter, and U. S. Kamilov, "Inference for generalized linear models via alternating directions and Bethe free energy minimization," arXiv:1501.01797, Jan. 2015.

[54] M. Vidyasagar, Nonlinear Systems Analysis. Englewood Cliffs, NJ: Prentice-Hall, 1978

[55] R. D. Yates, "A framework for uplink power control in cellular radio systems," IEEE J. Sel. Areas Comm., vol. 13, no. 7, pp. 1341-1347, September 1995. 